\title{
Implikationen für die Soziale Arbeit mit Flüchtlingen und Migranten
}

\author{
Monika Pfaller-Rott
}

Es folgen die Ergebnisse der internationalen und professionsübergreifenden Diskussion, welche im Rahmen der internationalen Tagung ,Social Diversity International Social Work from an Intercultural and De-Colonial Perspective“ zum Thema ,Implications for Social Work with Refugees and Migrants' erfolgte. Exemplarisch und länderspezifisch folgt eine Auseinandersetzung mit ausgewählten Aspekten zu Diversität, Gender, Rasse, Religion, Alter, Ability (psychische und physische Gesundheit), Schicht (sozialer Hintergrund), Kultur, Ethik, Erziehung, sexuelle Orientierung etc. Mit Respekt und Sensibilität in Bezug auf Unterschiede agiert Soziale Arbeit ressourcen-, coping- und vor allem auch forschungsorientiert.

Exemplarisch wurden die wissenschaftliche Debatte über Migrationsforschung (z.B. Binnenmigration, Immigration und Emigration) und deren Folgen für die Betroffenen selbst (Gender Diskussionen), für die Familien (, left behind families" / , left behind grandparents') und für die Gesellschaft (,economic migrants', ,interreligiöser Dialog', Versöhnungsprozesse) dargestellt. Aus dem reichhaltigen interkulturellen Erfahrungsschatz des friedlichen und respektvollen Miteinanders (mit indigener Bevölkerung, Afroamerikaner in Kolumbien, Dalits in Indien etc.) können wir unsere lokalen Lösungen vor Ort durch die Brille der anderen Länder reflektieren und daraus Lernprozesse initiieren. Globale Diskussionen verlangen nach globalen Bemühungen, deshalb legt auch eine Vertreterin der International Association of Schools of Social Work (IASSW) deren Sichtweise dar. 